\title{
3. RADIO REFLECTIONS FROM METEOR TRAILS
}

\author{
V. N. Lebedinec and A. K. Sosnova \\ (Astronomical Council, Academy of Sciences of \\ the U.S.S.R., Moscow)
}

\section{Reflection Coefficient}

Intensive development of the radar method of investigating meteors and connected phenomena in the Earth's atmosphere required studying interaction of radio waves with ionized meteor trails. Approximate solutions of the problem of radio reflection by ionized meteor trails have been obtained in a number of papers (Feinstein, 1950; Herlofson, 1951; Kaiser and Closs, 1952; Manning, 1953, 1963; Eshleman, 1955; Brysk, 1958, 1959).

The meteor trail is considered to be a narrow plasma cylinder of infinite length. A plane electromagnetic wave of random polarization falls on the cylinder normal to its axis. The trail expands radially under the action of ambipolar diffusion. Radio reflection is mainly from the electrons.

As a consequence of diffusion there is established a Gaussian distribution of electron density $N$ along the cylinder radius

$$
N=N_{0} \exp \left[-\left(\frac{r}{r_{0}}\right)^{2}\right]=\frac{\alpha}{4 \pi D\left(t+t_{0}\right)} \exp \left[-\frac{r^{2}}{4 D\left(t+t_{0}\right)}\right],
$$

where $r=$ distance from cylinder axis, $r_{0}=$ cylinder radius (the distance from the axis at which electron density is less by $1 / e), \alpha=$ electron-line density of the trail, $D=$ ambipolar diffusion coefficient, $t=$ time, $t_{0}=r_{H}^{2} / 4 D, r_{H}=$ initial trail radius.

The problem of radio reflections by an ionized cylinder can be reduced to a formal electrostatic problem (Stratton, 1941; Kaiser and Closs, 1952; Ginsburg, 1960). If the $x$-axis lies in the direction of incident wave propagation and the $y$-axis along the cylinder, then the incident electric vector $E=E_{0} e^{-i w t}$ and the magnetic one $H=H_{0} e^{-i w t}$ will be normal to the $x$-axis.

Complex permittivity of the cylinder is

$$
\varepsilon(r)=1-\frac{4 \pi e^{2}}{k^{2} m c^{2}} N(r)\left[1+i \begin{array}{c}
v \\
\omega
\end{array}\right],
$$

where $k=2 \pi / \lambda=$ wavenumber, $\lambda=$ wavelength, $e, m=$ electron charge and mass, $c=$ light velocity, $\omega=k c, v=$ frequency of electron collisions in the trail.

When the incident wave interacts with the cylinder there appears a reflected wave. 
Field inside the cylinder is described by Maxwell equations which reduce to equations

$$
\begin{array}{rlrl}
\operatorname{rot} \mathbf{H} & =-i \varepsilon k \mathbf{E} & & \operatorname{div} \mathbf{B}=0, \\
\operatorname{rot} \mathbf{E}=i k \mathbf{H} & & \operatorname{div} \mathbf{D}=0,
\end{array}
$$

where $\mathbf{D}=\varepsilon \mathbf{E}, \mathbf{B}=\mu \mathbf{H}, \mu=$ magnetic permeability (in our case $\mu=1$, Ginsburg, 1960). Equations for $\mathbf{E}$ and $\mathbf{H}$ are obtained from (3)

$$
\begin{gathered}
\operatorname{grad} \frac{1}{\varepsilon} \times \operatorname{rot} \mathbf{H}+{ }_{\varepsilon}^{1} \operatorname{rot} \operatorname{rot} \mathbf{H}=k^{2} \mathbf{H} \\
\operatorname{rot} \operatorname{rot} \mathbf{E}=\varepsilon k^{2} \mathbf{E}
\end{gathered}
$$

$\mathbf{E}$ and $\mathbf{H}$ can be found more easily in two particular cases: (1) electric field parallel, and (2) normal to the cylinder axis. Let us introduce $E_{\|}, H_{\perp}$ and $E_{\perp}, H_{\|}$, respectively. We shall solve the problem in cylindrical coordinates $r, \theta, z$.

In the case of parallel polarization $E=E_{\|}=E_{z}, E_{x}=E_{y}=0$, and determination of $E$ and $H$ reduces to the solution of the following equations:

$$
\begin{gathered}
\frac{\mathrm{d}^{2} P_{n}}{\mathrm{~d} \rho^{2}}+\frac{1 \mathrm{~d} P_{n}}{\rho \mathrm{d} \rho}+\left[\varepsilon\left(k r_{0}\right)^{2}-\frac{n^{2}}{\rho^{2}}\right] P_{n}=0 \\
E=E_{z}=\sum_{0}^{n} P_{n} \cos n \theta, \quad H=H_{\theta}=-\frac{1}{i k} \sum_{0}^{n} P_{n}^{\prime} \cos n \theta, \quad \rho=\frac{r}{r_{0}}, \quad n=0, \pm 1, \pm 2, \ldots
\end{gathered}
$$

In the case of transverse polarization $H=H_{\|}=H_{z}, H_{x}=H_{y}=0$, and determination of $\mathbf{E}$ and $\mathbf{H}$ reduces to the solution of the following equations:

$$
\begin{aligned}
& \left.\frac{\mathrm{d}^{2} T_{n}}{\mathrm{~d} \rho^{2}}+\left(\begin{array}{c}
1 \\
\rho
\end{array}-{ }_{\rho}^{\varepsilon_{\rho}^{\prime}}\right)^{2}\right) \frac{\mathrm{d} T_{n}}{\mathrm{~d} \rho}+\left[\varepsilon\left(k r_{0}\right)^{2}-\begin{array}{c}
n^{2} \\
\rho^{2}
\end{array}\right] T_{n}=0, \\
& H=H_{z}=\sum_{0}^{n} T_{n} \cos n \theta, \quad E=E_{\theta}=\frac{1}{i \varepsilon k} \sum_{0}^{n} T_{n}^{\prime} \cos n \theta .
\end{aligned}
$$

The corresponding reflection coefficients $A_{\|}$and $A_{\perp}$ are determined from the boundary conditions for $\varepsilon=1$ :

$$
\begin{aligned}
& A_{\|}=\sum_{0}^{n} \tau \frac{P_{n}^{\prime} J_{n}-k J_{n}^{\prime} P_{n}}{-H_{n}^{(1)} P_{n}^{\prime}+k{H_{n}^{\prime(1)}}_{n}} \cos n \theta, \\
& A_{\perp}=\sum_{0}^{n} \tau \frac{J_{n} T_{n}^{\prime}-k J_{n}^{\prime} T_{n}}{-H_{n}^{(1)} T_{n}^{\prime}+k H_{n}^{\prime(1)} T_{n}} \cos n \theta,
\end{aligned}
$$

where $\tau=1$ for $n=0, \tau=2$ for $n \neq 0, J_{n}=J_{n}(k \rho)=$ the Bessel function of the first kind, $H_{n}^{(\mathrm{l})}=J_{n}(k \rho)+i N_{n}(k \rho)=$ the Hankel function of the first kind, $\theta=180^{\circ}$. Total reflec- 
tion coefficients $g_{\|}$and $g_{\perp}$ are determined as moduluses of complex values $A_{\|}$and $A_{\perp}$ (Morse and Feshbach, 1953).

Equations (6) and (7) are not solved analytically. The behaviour of functions $P$ and $T$ as well as $g_{\|}$and $g_{\perp}$ was investigated at various limitations by Lovell and Clegg (1952). It was shown by Herlofson (1951) that in case of $r_{0} \ll \lambda$ and electron density close to critical one, the phenomenon of resonance was possible, that is the ratio $g_{\perp} / g_{\|}$sharply increased. For a trail with low electron-line density $\alpha \ll 2.4 \times 10^{12}$ $\mathrm{cm}^{-1}$ (so-called underdense trails) reflection coefficients were determined under the assumption that the reflected wave was a superposition of partial waves scattered independently and coherently by electrons (Herlofson, 1951; Kaiser and Closs, 1952; Lovell and Clegg, 1952).

For trails with $\alpha<2.4 \times 10^{12} \mathrm{~cm}^{-1}$ and $\left|\varepsilon\left(k r_{0}\right)^{2}\right|<1$ Kaiser and Closs (1952) obtained values of $g_{\|}$and $g_{\perp}$ using the approximate numerical solution of Equations (6) and (7) for $n=1$ and $n=0$. The ratio $g_{\perp} / g_{\|}$reached the maximum value $g_{\perp} / g_{\|}=2$ when $\varepsilon=-1.4$ on the cylinder axis. For trails with $\alpha>2.4 \times 10^{12} \mathrm{~cm}^{-1}$ (so-called overdense trails) the assumption of scattering by individual electrons is unusable. In this case, for $\left|\varepsilon\left(k r_{0}\right)^{2}\right| \gg 1$, Kaiser and Closs (1952), Eshleman (1955) and others determine reflection coefficients supposing that the trail reflects radio waves like a metallic cylinder. The effect of radio-wave refraction in the outer cylinder parts was taken into account by Manning (1953, 1963). Kaiser and Closs (1952) obtained curves of $g_{\|}$and $g_{\perp}$ change in the transitional area $\alpha \approx 10^{12} \mathrm{~cm}^{-1}$ by joining smoothly the two solutions for ranges $\alpha<2.4 \times 10^{12} \mathrm{~cm}^{-1},\left|\varepsilon\left(k r_{0}\right)^{2}\right|<1$ and $\alpha>2.4 \times 10^{12} \mathrm{~cm}^{-1},\left|\varepsilon\left(k r_{0}\right)^{2}\right| \gg 1$.

Exact values of $g_{\|}$and $g_{\perp}$ for $\alpha=10^{11} \mathrm{~cm}^{-1}$ in the range of $k r_{0}=0.01$ to 1 and for $\alpha=10^{15} \mathrm{~cm}^{-1}$ in the range of $k r_{0} 0 \cdot 1$ to 2 were obtained by Keitel (1955) by numerical integration of Equations (6) and (7). It was assumed that $v / \omega=10^{-4}$. The results obtained confirmed the validity of the electron-scattering model. for $g_{\|}$at $\alpha=10^{11} \mathrm{~cm}^{-1}$ and of the metallic cylinder model at $\alpha=10^{15} \mathrm{~cm}^{-1}$. At $\alpha=10^{11} \mathrm{~cm}^{-1}$ the existence was confirmed of a resonance maximum found by Kaiser and Closs when they analysed the behaviour of $g_{\perp}$ on the basis of an approximate numerical solution of Equation (7) for $n=1$. However, preliminary estimates of $g_{\|}$and $g_{\perp}$ at $\alpha=10^{13} \mathrm{~cm}^{-1}$ point to the existence of a small resonance $\left(g_{\perp} / g_{\|}\right.$is of the order of 1.5). This is inconsistent with the assumption made by Kaiser and Closs of no resonance in the case of trails with $\alpha>2.4 \times 10^{12} \mathrm{~cm}^{-1}$. At present, mainly meteors with $\alpha=10^{11}$ to $10^{13} \mathrm{~cm}^{-1}$ are recorded by the radar method. In this connection it is very interesting to determine more exactly the limits in which the Kaiser and Closs theory is applicable and to investigate the behaviour of $g_{\|}$and $g_{\perp}$ in the transitional region between underdense and overdense trails. For this purpose we numerically integrated Equations (6) and (7) with the help of an electronic computer 'Minsk-2' and computed $g_{\perp}$ and $g_{\|}$values for various $\alpha$ in the range $10^{10}-10^{13} \mathrm{~cm}^{-1}$ and $k r_{0}$ in the range 0.001 to 4 at two $v / \omega$ values: $10^{-4}$ and 0.07 (which correspond to the height range 
80-100 km and wavelength range 4-16 m) including orders $n$ from 0 to 7 . The RungeKutta method was used. Integration was performed with automatic choice of step. The Bessel functions $J_{n}(k \rho)$ of the first kind are taken as initial conditions for $P_{n}$ and $T_{n}$. The values of $P_{n}$ and $T_{n}$ were computed from $10^{-7}$ to 6 . The step was $0 \cdot 1$. In this range the program ensured exactness of integration to $10^{-4}$.

Equations (7) in the range $\varepsilon=0$ have a discontinuity which is however avoided during integration owing to a finite value of $v / \omega$, but the integration step here becomes small.

Reflection coefficients for parallel and transverse polarization $g_{\|}$and $g_{\perp}$ were computed with the help of Equations (8) and (9) for $\varepsilon=1-10^{-6}$ ( $\rho$ values in this case reached 3-5 depending on $\alpha$ and $k r_{0}$ ).

Figures 1-6 give curves of $g_{\|}, g_{\perp} / g_{\|}, \varphi_{\|}$and $\varphi_{\perp}\left(\varphi=\operatorname{arc} \operatorname{tg} A_{\text {im }} / A_{\text {re }}\right.$, where $A_{\text {im }}$ and $A_{\text {re }}$ are imaginary and real parts of $A$ ) as a function of $k r_{0}$ for two $v / \omega$ values: $10^{-4}, 0.07$ and for a number of $\alpha$ values; $(0)-10^{10},(1)-5 \times 10^{10},(2)-2 \times 10^{11}$, (3) $-5 \times 10^{11},(4)-10^{12},(5)-2 \times 10^{12},(6)-3 \times 10^{12},(7)-5 \times 10^{12},(8)-10^{13} \mathrm{~cm}^{-1}$. Curves (Figures 1-2) calculated according to the formulas for underdense trails with $\alpha \ll 2 \times 10^{12} \mathrm{~cm}^{-1}$ (Kaiser and Closs 1952; Kaiser, 1955) are drawn by the broken line. Curves (9) for a metallic cylinder with the radius $k r_{0}$ are also given for comparison (Frank and Mises, 1930-35; King and Wu, 1959). Figure 7 shows the maximum value of $g_{\perp} / g_{\|}$as a function of $\alpha$.

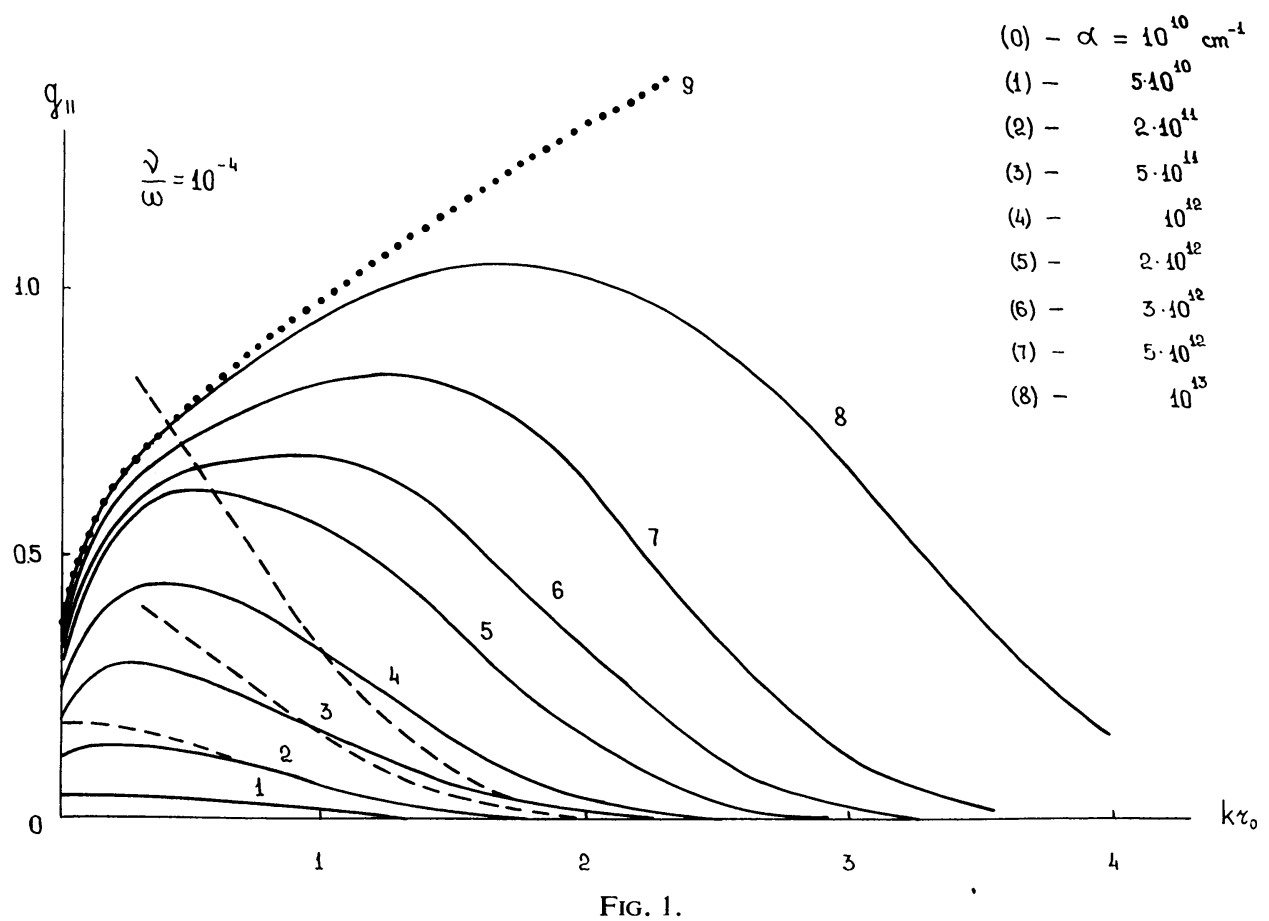




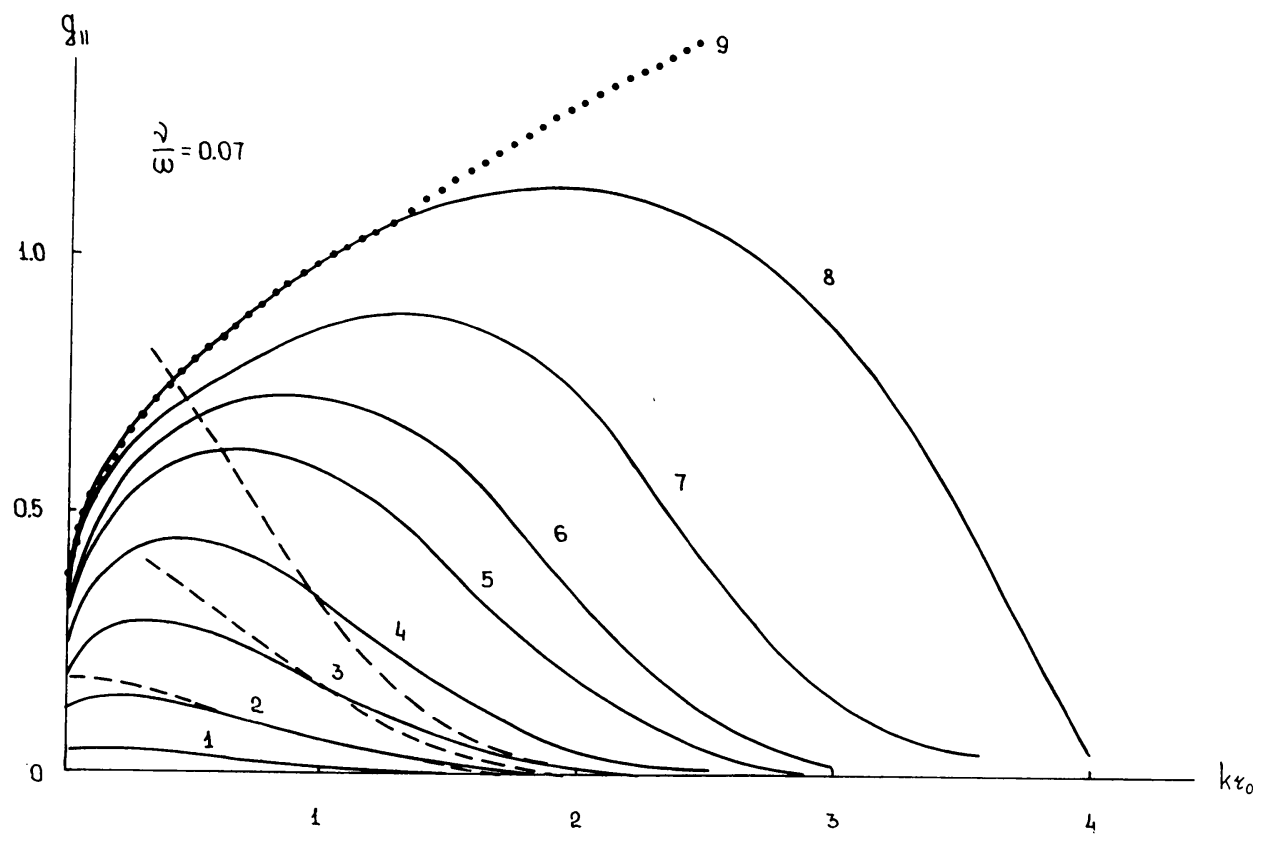

FIG. 2.

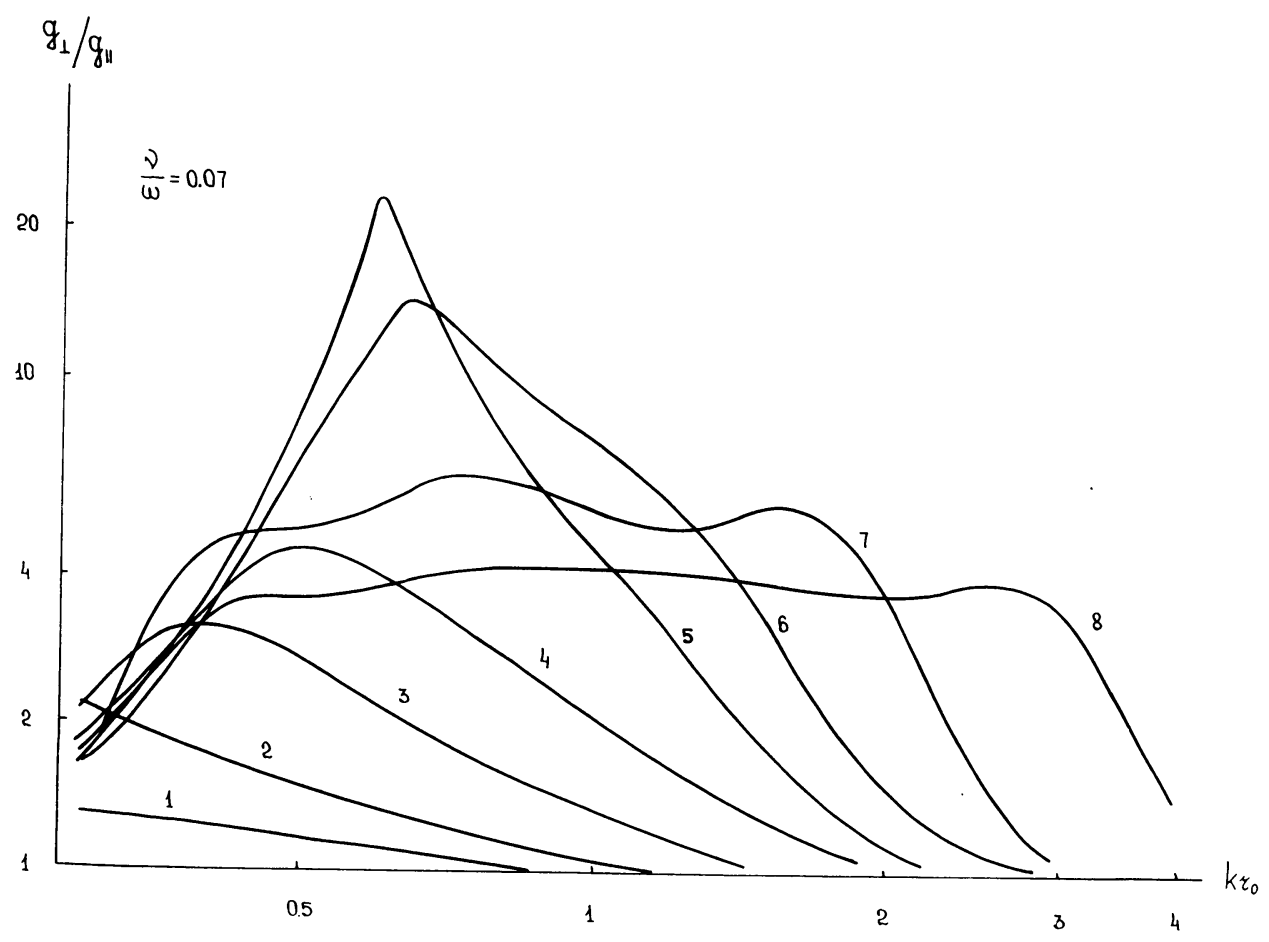

FIG. 3. 


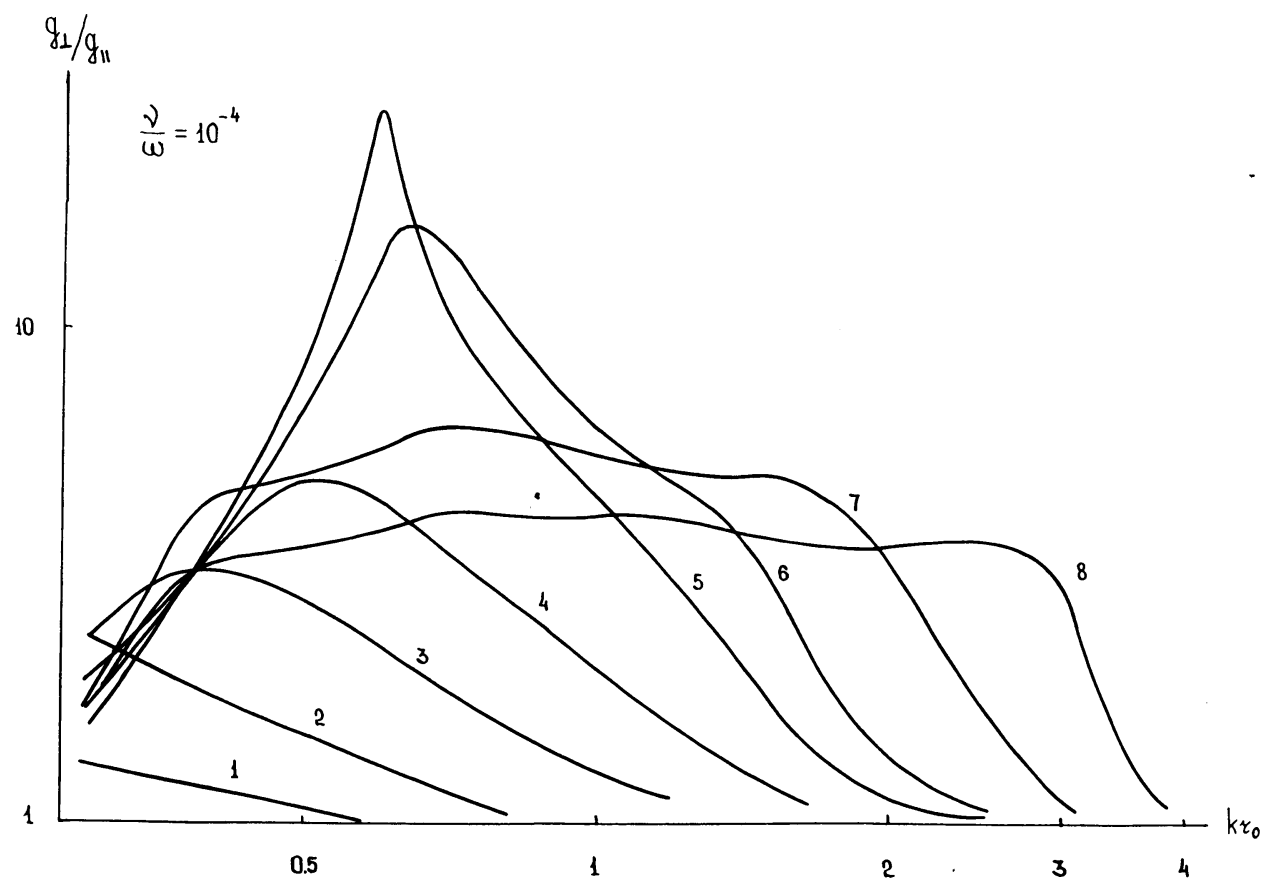

FIG. 4.

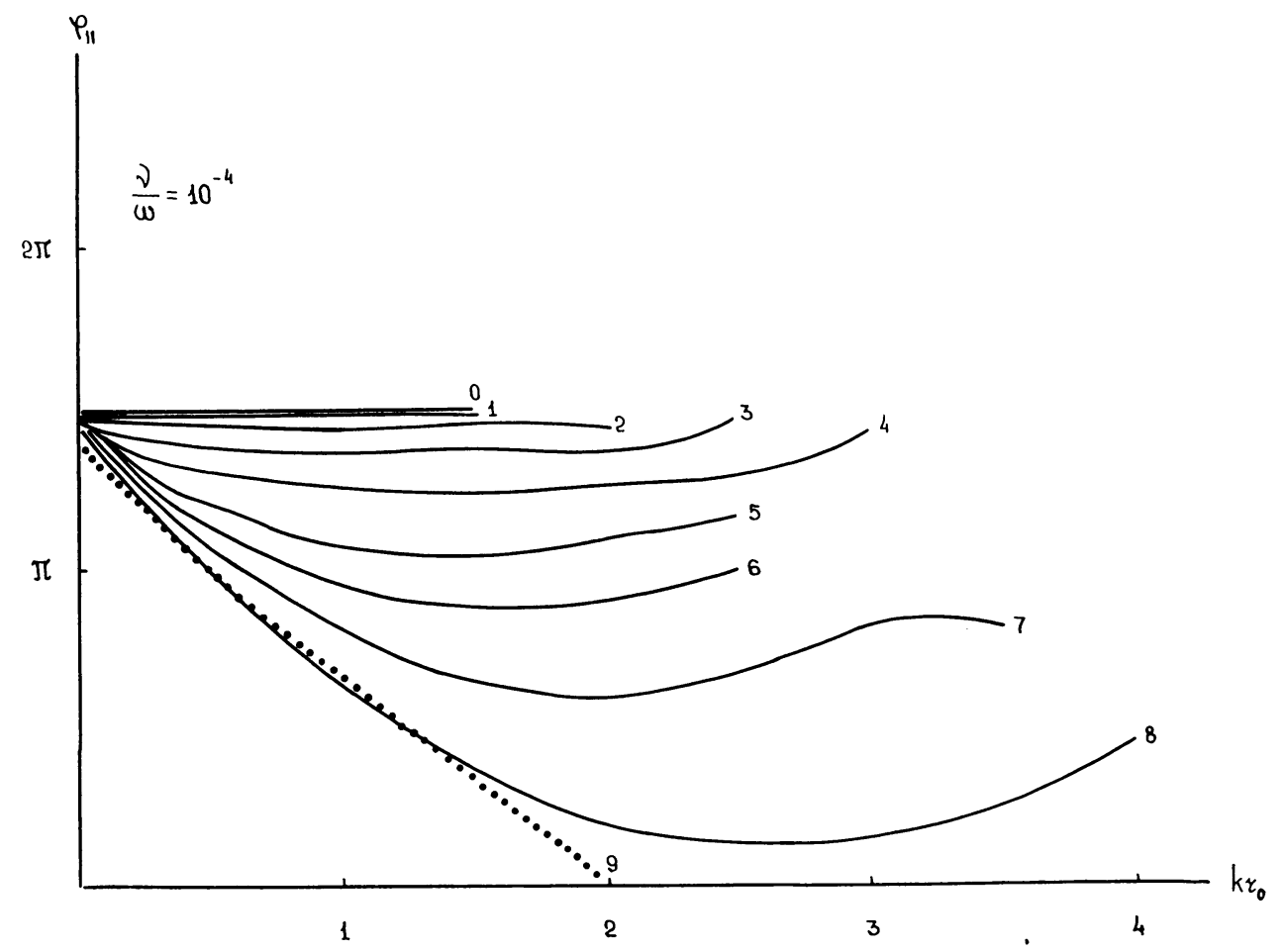

FIG. 5 . 


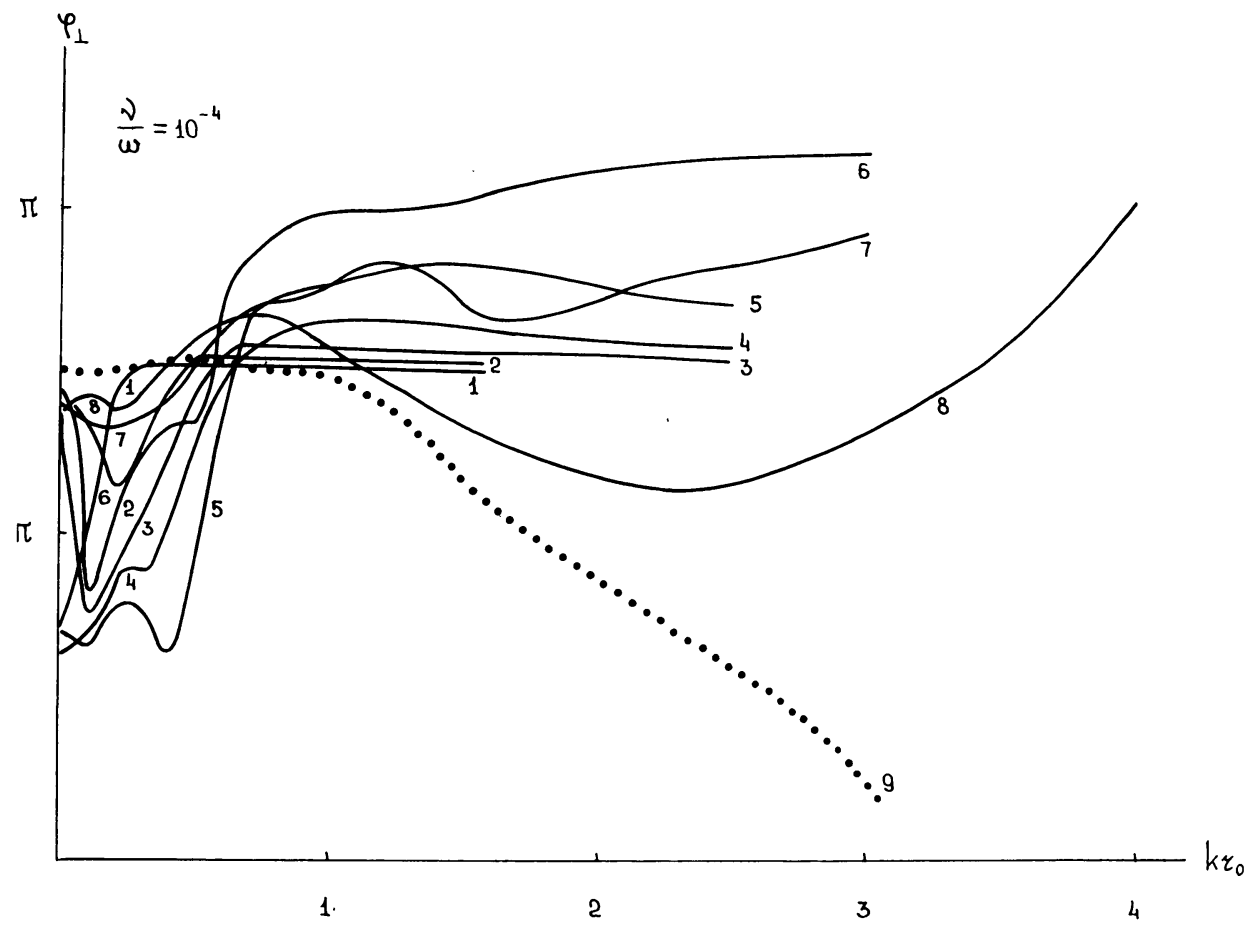

FIG. 6.

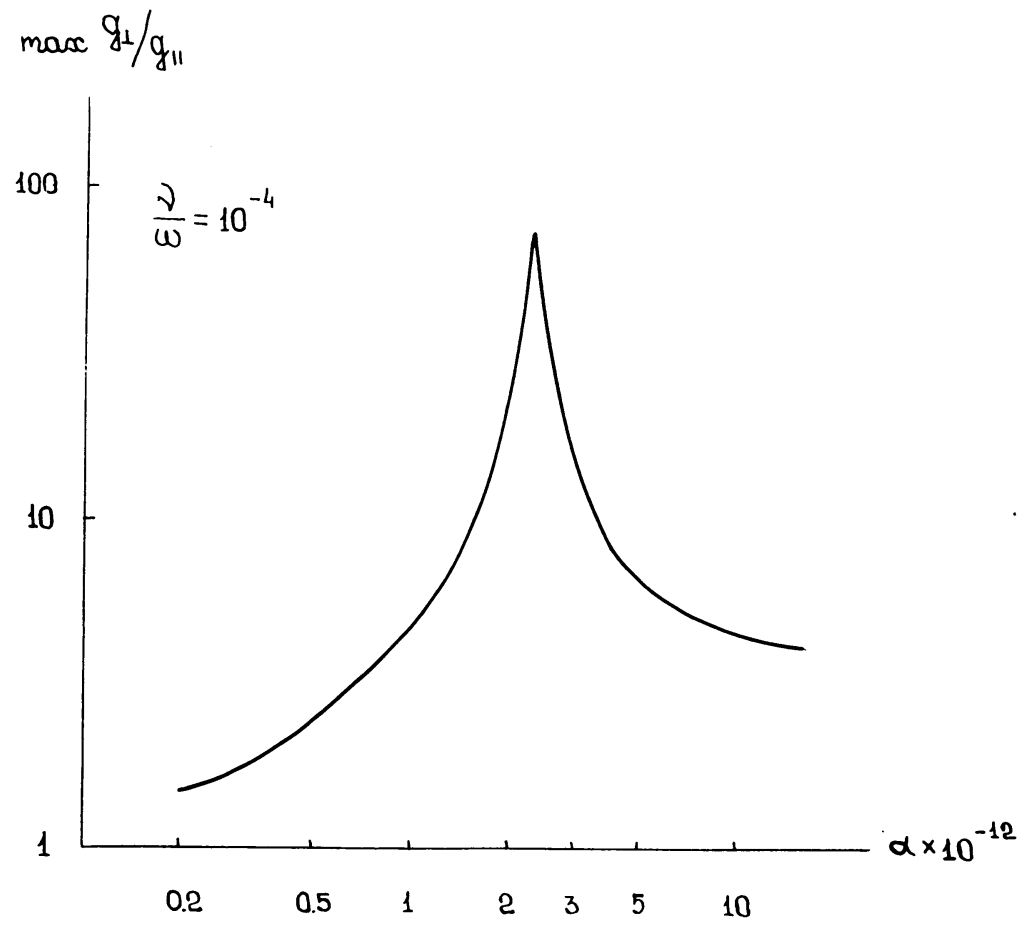

FIG. 7. 
As it is seen from the figures, curves $g_{\|}$and $g_{\perp} / g_{\|}$for two chosen $v / \omega$ values practically coincide. Calculated values of $g_{\perp}$ and $g_{\|}$give a sharp resonance peak in that range of $\alpha$ and $k r_{0}$ change where $\varepsilon=-1.4$ on the cylinder axis at $\alpha$ close to $2 \cdot 3 \times 10^{12} \mathrm{~cm}^{-1}$. Resonance does not disappear at $\alpha>2.4 \times 10^{12} \mathrm{~cm}^{-1}$ and $\left(k r_{0}\right)^{2}>1$, and $g_{\perp} / g_{\|}$value can considerably exceed 2 . This contradicts the Kaiser and Closs conclusions that resonance disappears at $\alpha>2.4 \times 10^{12} \mathrm{~cm}^{-1}$ and $\left(k r_{0}\right)^{2}>1$, and that the $g_{\perp} / g_{\|}$value does not exceed 2 in the case of a Gaussian distribution of the electron concentration along the cylinder radius. Kaiser and Closs ascribed the values of resonance ratio $g_{\perp} / g_{\|}$higher than 2 observed by Clegg and Closs (1951) to the anisotropy of ambipolar diffusion coefficient $D$ caused by the magnetic field of the Earth. The exact solution of the problem shows that resonance ratio $g_{\perp} / g_{\|}$can exceed 2 for trails with $\alpha=10^{12}-10^{13} \mathrm{~cm}^{-1}$, even under isotropic diffusion.

Resonance appearance in case of slightly overdense trails and those of a transitional kind is confirmed by observational results (Billam and Browne, 1955; Sidorov et al., 1965).

\section{Diffraction Patterns}

The amplitude of the signal reflected by a meteor trail (Kaiser, 1955; Kaščeev and Lebedinec, 1961) is

$$
E_{r}=\frac{G \lambda \sqrt{2 R_{b x}}}{4 \pi R^{2}} \int_{-\infty}^{s_{0}} g e^{i \frac{4 \pi R}{i}} \mathrm{~d} s
$$

where $g(s)=$ reflection coefficient for the given trail point, $s=$ distance from the specular point on the trail to the given point along the trail axis reckoned in the direction of meteor movement; $s_{0}=$ coordinate $s$ for the trail head; $G=$ antenna gain; $R_{b x}=$ input resistance of the receiver; and $R=$ distance from radar to the given point on the trail.

Trail parts close to the specular point for which $s \ll R$ and $R$ changes very little, contribute mostly to $E_{r}$. Therefore one can approximate

$$
R=\sqrt{R_{0}^{2}+s^{2}}=R_{0}+\frac{1}{2} \frac{s^{2}}{R_{0}^{2}},
$$

where $R_{0}$ is a distance from radar to the specular point. Assuming that

we find

$$
x=\frac{2 s}{\sqrt{R_{0} \lambda}} \text { and } b=\frac{G \lambda \sqrt{2 R_{b x}} \sqrt{R_{0} \lambda}}{4 \pi R_{0}^{2}} e^{i \frac{4 \pi R_{0}}{\lambda}}
$$

$$
E=b \int_{-\infty}^{x_{0}} g e^{i x^{2 x^{2}}} \mathrm{~d} x
$$


Here $g=g\left(k r_{0}\right),\left(k r_{0}\right)^{2}=\left(k r_{H}\right)^{2}+\Delta\left(x_{0}-x\right), \Delta=\left(8 \pi D \sqrt{ } R_{0}\right) /\left(v \lambda^{3 / 2}\right), v$ is meteor velocity.

For underdense trails in the case of parallel polarization according to Kaiser (1955)

$$
g=\pi \alpha\left(\frac{e^{2}}{m c^{2}}\right) e^{-k^{2} r_{0}^{2}}
$$

Then (Kaščeev and Lebedinec, 1961)

$$
E_{r}=\frac{\sqrt{R_{0} \lambda}}{2}\left(2 R_{b x} W_{0}\right)^{1 / 2} \int_{-\infty}^{x_{0}} \alpha e^{-\left(\begin{array}{c}
2 \pi r_{0} \\
\lambda
\end{array}\right)^{2}} e^{-\Delta\left(x_{0}-x\right)} e^{\mathrm{i} \pi x^{2}} \mathrm{~d} x
$$

where

$$
W_{0}=\frac{G^{2} \lambda^{2}}{16 \pi^{2} R^{4}}\left(\begin{array}{c}
e^{2} \\
m c^{2}
\end{array}\right)^{2}
$$

and the power of the echo from all the trail is

$$
W=\frac{G^{2} \lambda^{3} \alpha^{2}}{32 \pi^{2} R^{3}}\left(\frac{e^{2}}{m c^{2}}\right)^{2} e^{-2\left(\begin{array}{c}
2 \pi r_{H} \\
\lambda
\end{array}\right)^{2}} J^{2},
$$

where

$$
J=\mid \frac{1}{\sqrt{2}} \int_{-\infty}^{x_{0}} e^{\mathrm{i} \frac{\pi x^{2}}{2}} e^{-\Delta\left(x_{0}-x\right)} \mathrm{d} x
$$

Positions of diffraction pattern maxima and minima calculated from (13) can be used for meteor velocity determination.

It was Herlofson who pointed first to the possibility of meteor-velocity measurements from observations of Fresnel diffraction of radio waves by meteor trails (Lovell and Clegg, 1952). This idea was developed by Davies and Ellyett (1949). Theoretical diffraction patterns $E\left(x_{0}\right)$ for the case of parallel polarization were obtained by Kaiser (1953) by numerical integration. Values of reflection coefficients presented by Kaiser and Closs (1952) were used. Figure 5 of Kaiser (1953) shows qualitatively the change in $E\left(x_{0}\right)$ for various $\Delta$ values. For underdense trails Brysk (1958) derived analytical expressions for $E\left(x_{0}\right)$ and diffraction patterns from them. A table of positions of diffraction pattern maxima was also given. Kaščeev and Lebedinec (1961) numerically integrated Equation (15) and compared for the first time calculated results with observed diffraction patterns for underdense trails.

Theoretical diffraction patterns derived by Kaščeev and Lebedinec (1961) are close to the results of Brysk (1958). Tables for $v$ determination were made by Davies and Ellyett (1949) and in more details by Simek (1964), the effect of trail-diffusion expansion being left out of account. Simek (1966) calculated diffraction patterns of the echo, taking into account diffusion effect for a great number of $\Delta$ values. But ap- 
parently some mistakes were made in calculations, which results, for example, in the fact that transition from the specular reflection to the head echo did not occur with increase in $\Delta$. This transition, according to Brysk (1958) and Kaščeev and Lebedinec (1961) occurs at $\Delta \approx 2$. Positions of the first maximum of diffraction patterns in Table I of Šimek (1966) are also erroneous at $\Delta \geqslant 0.5$.

By numerical integration we obtained the value of

$$
E\left(x_{0}\right)=\int_{-\infty}^{x_{0}} g e^{i^{\pi x^{2}}} 2 \mathrm{~d} x
$$

for parallel $\left(g=g_{\|}\right)$and transverse $\left(g=g_{\perp}\right)$ scattering at $0<x_{0}<4 \cdot 10$ for a number of $\Delta$ and $k r_{H}$ values in the ranges $0<\Delta<4$ and $0<k r_{H}<2$. The Gaussian method was used. Tables of $I$ values were calculated in the same ranges of $x_{0}$ and $\Delta$.

Theoretical diffraction patterns obtained in such a way for trails which are underdense or of a transitional type at parallel scattering (cf. Figures 8-10, where 1, 2, 3, 4,5 , show the diffraction curves at $\Delta$ values: $0 \cdot 001,0 \cdot 5,1 \cdot 0,1 \cdot 5$ and $2 \cdot 0$, respectively) confirmed conclusions drawn by Brysk, and Kaščeev and Lebedinec, that the first maximum of the diffraction pattern is noticeably shifted with increasing $\Delta$ and positions of the other extremes changed considerably less. Conclusions made by Kaščeev and Lebedinec (1961) that at $\Delta \geqslant 2$ the diffraction pattern disappears and only the head echo is observed, are also confirmed. The obtained curves show that it is possible to use Equation (15) for velocity calculations for the case of trails which are underdense or of a transitional type up to $\alpha=10^{12}$ at parallel scattering. The error does not exceed 2 or $3 \%$ and is of a random character. At transverse scattering and $\alpha \gtrsim 5 \times 10^{11}$, as a result of resonance, the errors in velocity calculations increase and can sometimes reach $6-12 \%$ (cf. Tables $1,2,3$ ). In these tables distances between the various extremes of diffraction curves calculated from (15) are denoted by $A C_{k k}$, $A E_{k k}$, and so on, and those calculated from (11) are denoted by $A C, A E$, and so on.

An initial radius of ionized meteor trails can be calculated with the help of (15) from results of simultaneous radar observations of the same underdense trails at two different wavelengths $\lambda_{1}, \lambda_{2}$ (Kaščeev and Lebedinec, 1961). Diffraction patterns obtained by us allow evaluation of the error in determination of an initial radius $r_{H}$ by this method if trails of a transitional type are observed. Figure 11 shows $r_{H \text { cal }} / r_{H \text { in }}$ as a function of $\alpha$ for a number of $k r_{H}$ values. Here $r_{H \text { cal }}$ denotes the value of an initial radius, $r_{H}$, calculated according to the method described by Kaščeev and Lebedinec (1961), $r_{H \text { in }}$ denotes the true value of $r_{H}$.

From Figure 11 it is seen that already at $\alpha=5 \times 10^{11} \mathrm{~cm}^{-1}$ the $r_{H}$ value determined from simultaneous radar observations of the same trails at two wave-lengths can be underestimated by $30-70 \%$. This result was predicted by Lebedinec (1963). Diffraction patterns of echoes from trails of a transitional type do not differ in appearance from 

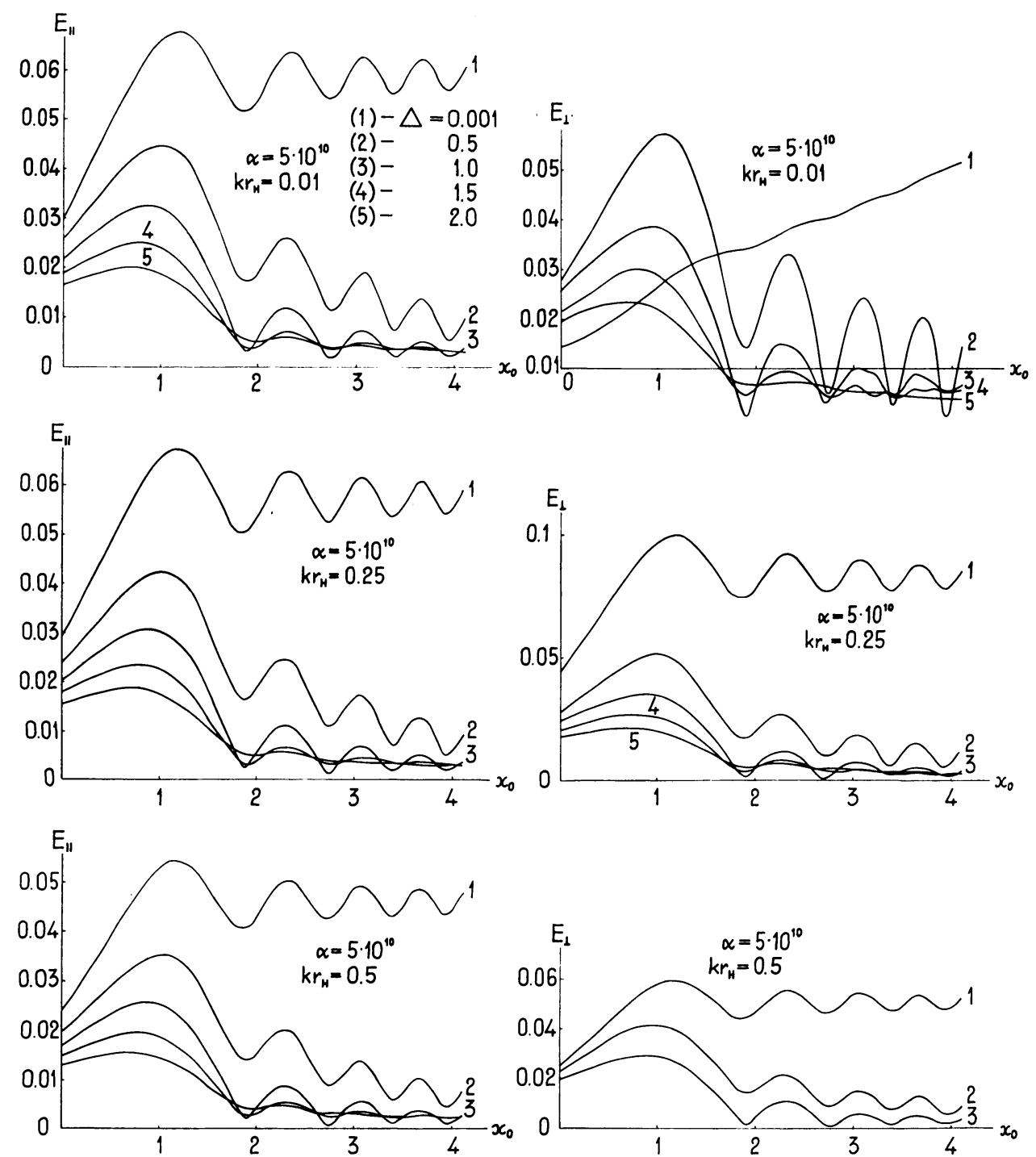

Fig. 8. 

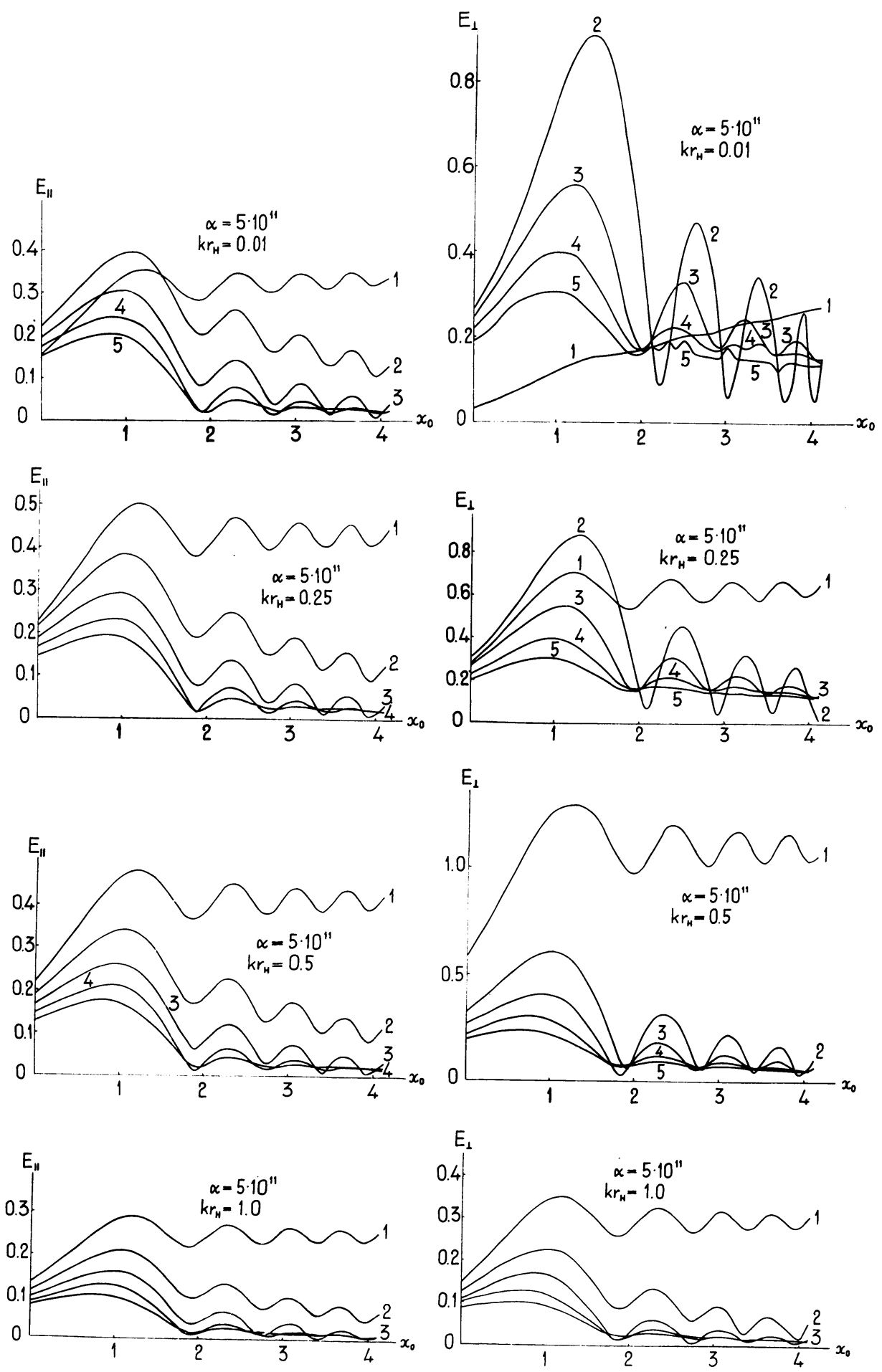

FIG. 9. 

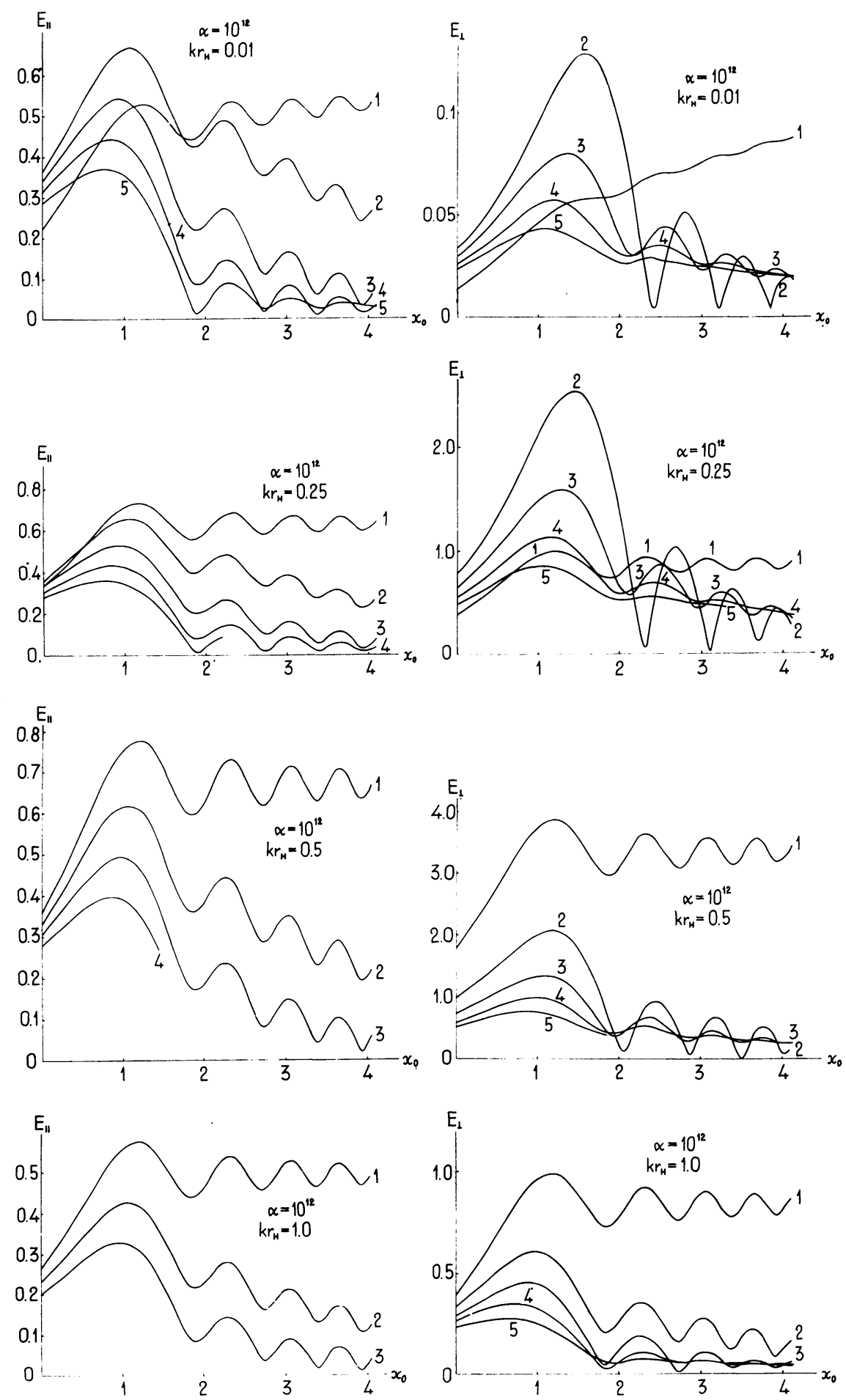

FIG. 10 . 


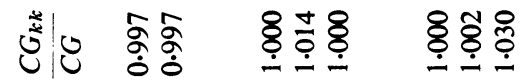

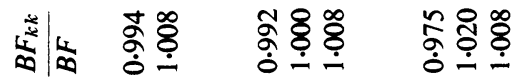

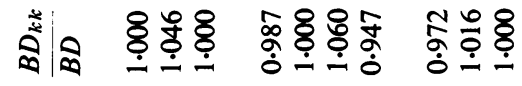

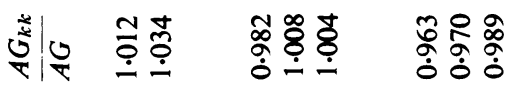

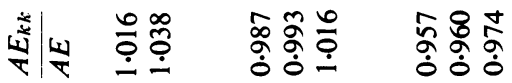

ฟैำ

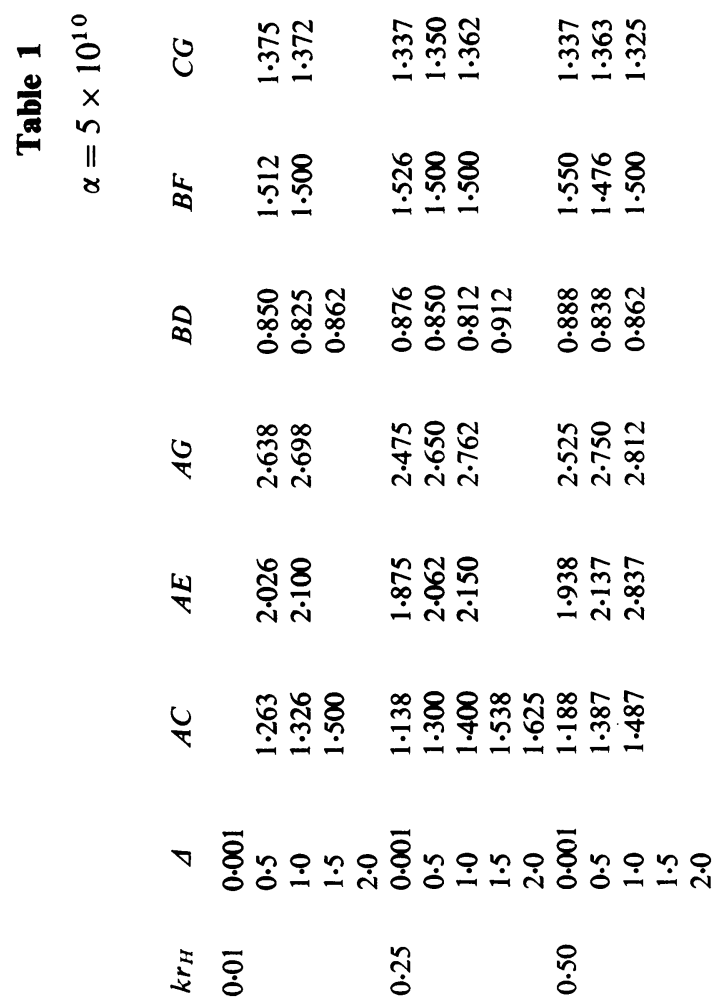




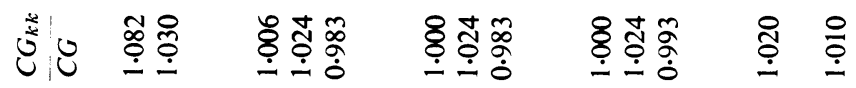

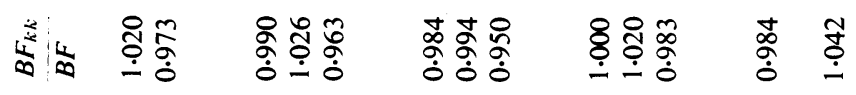

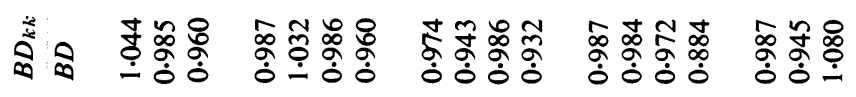

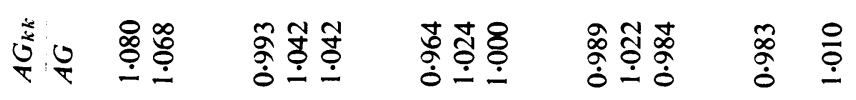

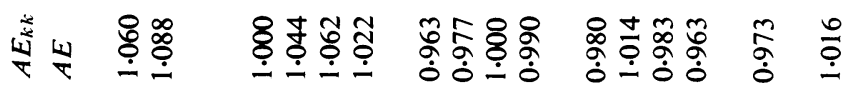

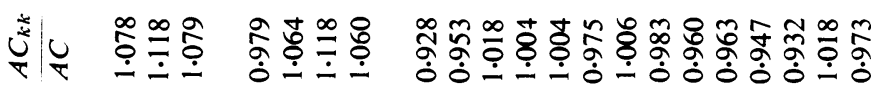

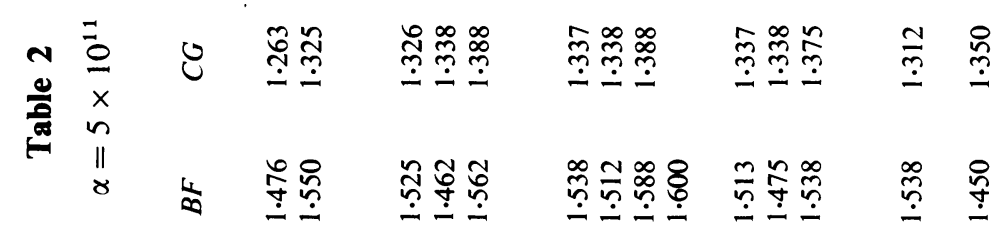

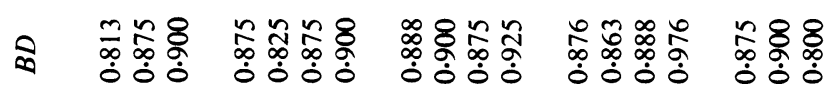

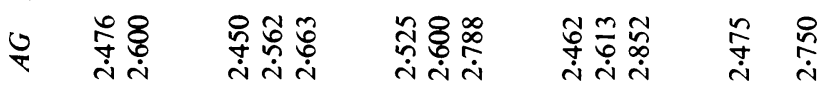

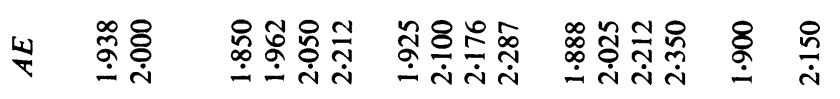

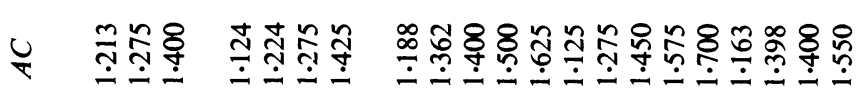

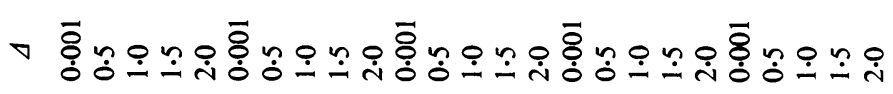

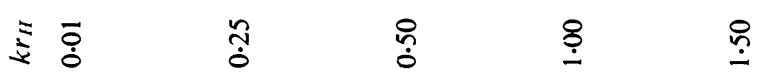




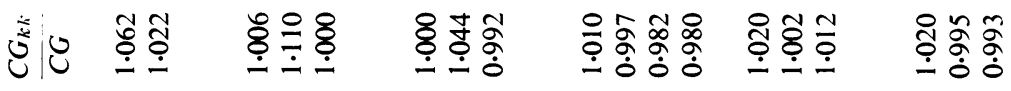

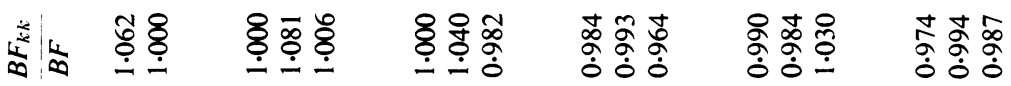

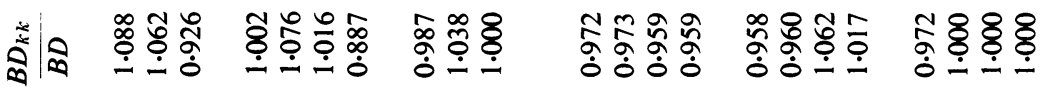

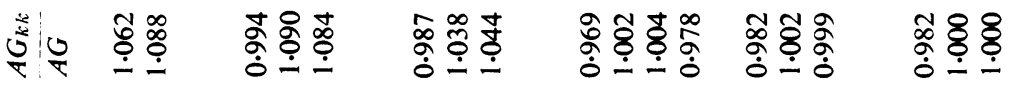

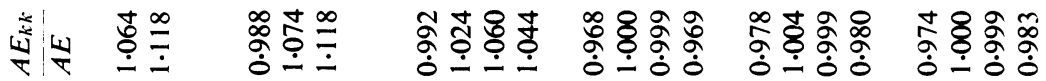

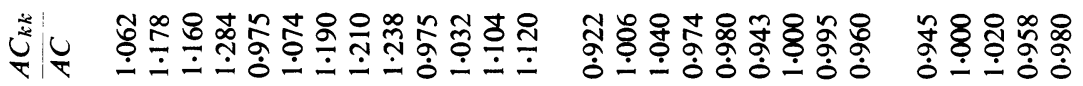
蛋

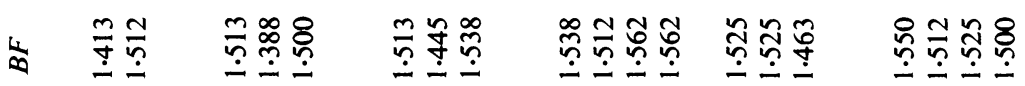

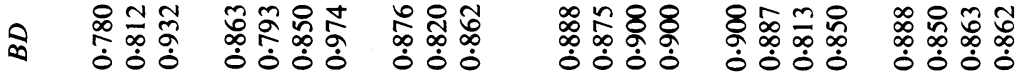

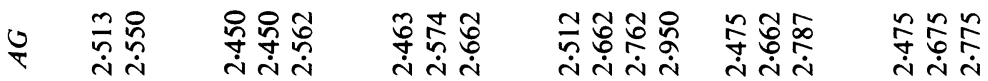

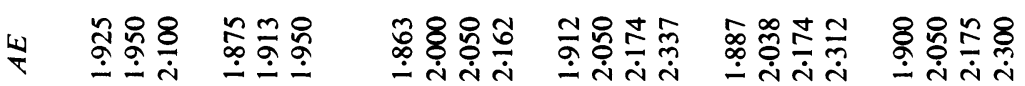

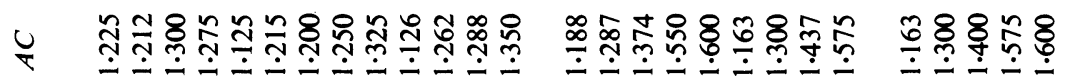

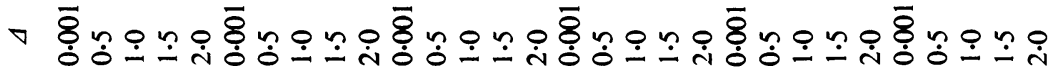

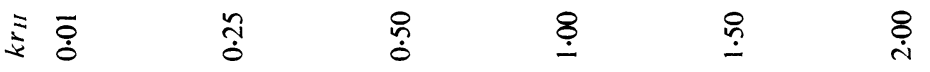




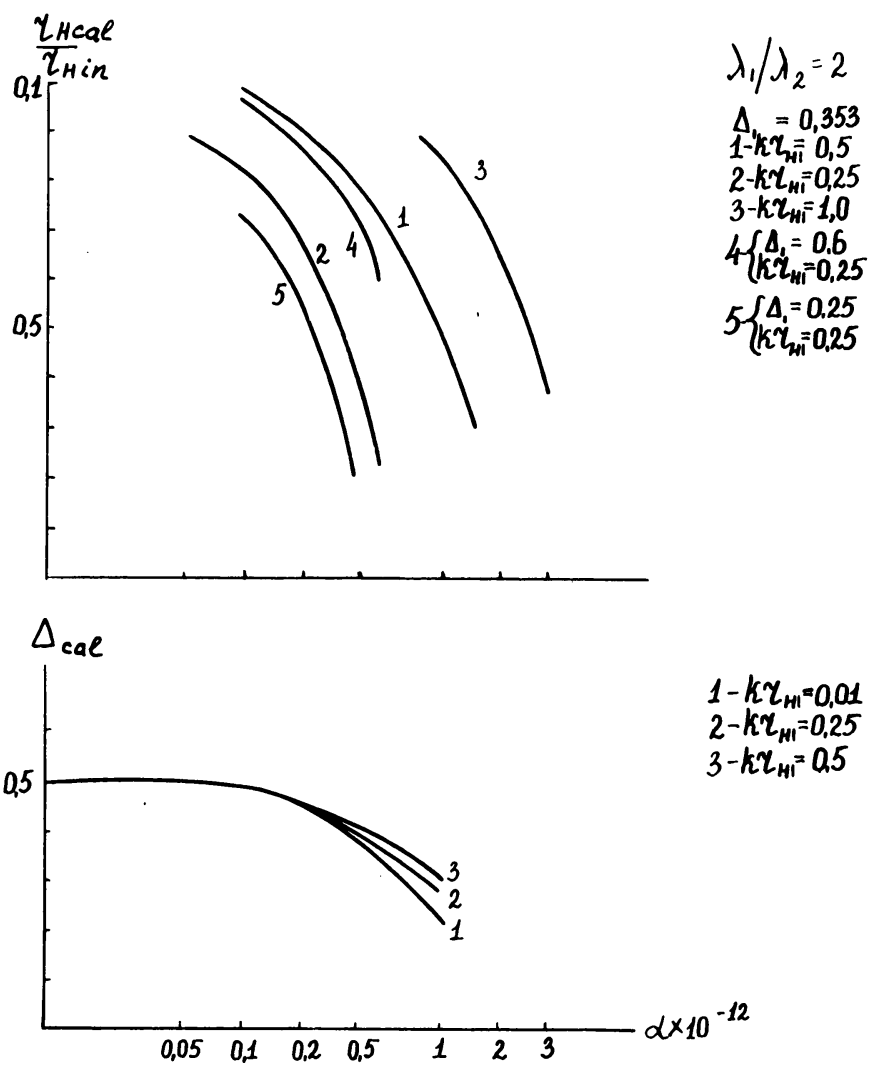

FIG. 11 .

those of echoes from underdense trails at parallel scattering. Approximately exponential time decrease of radio-echo amplitude from underdense trails, which follows from Equation (15), is often used for determination of ambipolar diffusion coefficient $D$ and atmospheric density $\rho_{\mathrm{a}}$. Figure 11 represents $\Delta_{\text {cal }}$ values defined according to exact diffraction patterns by finding the exponent which is in best agreement with $E$ time variations, values $\alpha, k \mu_{H}$ being different and $\Delta$ true value being $0 \cdot 5$. It is evident that with $\alpha>10^{11} \mathrm{D}$ values determined from observations are underestimated and $\rho_{\mathrm{a}}$ is overestimated. Thus, trails of a transitional kind with $\alpha>10^{11}$ should not be used for determination of $D$ and $\rho_{\mathrm{a}}$. So far only trails with $\alpha>10^{11}$ were used for this purpose. The same calculations were made by us for a large quantity of values $\alpha, \Delta$ and $k \mu_{H}$. It follows that value $\Delta$ can be estimated most precisely from observations of trails with $\alpha<10^{11}$ when $0 \cdot 2<\Delta<0 \cdot 8$. Using trails of a transitional kind, while determining ambipolar diffusion coefficient $D$ from exponential decrease of echo amplitude with time, can lead to considerable underestimation of the value of $D$. 


\section{References}

Billam, E. H., Browne, I.C. (1955) Astr. Contr. Univ. Manchester, Ser. II., 127.

Brysk, H. (1958) J. geophys. Res., 63, 693.

Brysk, H. (1959) I.R.E. Trans., AP-7, S 330.

Clegg, J.A., Closs, R.G. (1951) Proc. phys. Soc., London, B64, 718.

Davies, J.G., Ellyett, C.D. (1949) Phil. Mag., 40, 614.

Eshleman, V.R. (1955) I.R.E. Trans., AP-3, 32.

Feinstein, J. (1950) J. geophys. Res., 56, 37.

Frank, F., Mises, R. (1930-35) Differential and Integral Equations of Mathematical Physics, F. Vieweg und Sohn, Braunschweig.

Ginsburg, V.L. (1960) Electromagnetic Wave Propagation in Plasma, Gosudarstvennoe Izdatel'stvo, Moscow.

Herlofson, N. (1951) Ark. Fys., 3, 247.

Kaiser, T.R. (1953) Adv. Phys., 2, 495.

Kaiser, T.R. (1955) J. atmos. terr. Phys., Spec. Suppl., $2,55$.

Kaiser, T.R., Closs, R.L. (1952) Phil. Mag., 43, 1.

Kaščeev, B.L., Lebedinec, V.N. (1961) Rezultaty Issled. MGG - Ionosfera i Meteory, 7, 1.

Keitel, G.H. (1955) Proc. I.R.E., 1481.

King, R., Tai Tsun Wu (1959) The Scattering and Diffraction of Waves, Harvard University Press, Cambridge, Mass.

Lebedinec, V.N. (1963) Astr. Zu., 40, 719.

Lovell, A.C. B., Clegg, J.A. (1952) Radio Astronomy, Chapman and Hall, London.

Manning, L.A. (1953) J. atmos. terr. Phys., 4, 219.

Manning, L.A. (1963) J. atmos. terr. Phys., 25, 182.

Morse, P.M., Feshbach, H. (1953) Methods of Theoretical Physics, McGraw-Hill, New York.

Sidorov, V.V., Pavlov, A.F., Fakhrutdinov, R.J. (1965) Radiophysics, 8, 234. Izvestija vy̌šsih učebnyh zavedenij, S.S.S.R.

Stratton, J.A. (1941) Electromagnetic Theory, McGraw-Hill, New York.

Simek, M. (1964) A Table for the Calculations of Meteor Velocities, Astr. Inst. Csl. Acad. Sci., Ondřejov.

Simek, M. (1966) Bull. astr. Inst. Csl., 17, 354. 